\title{
Non-invasive measurement of coronary flow reserve in children with Kawasaki disease
}

\author{
N Noto, K Karasawa, H Kanamaru, M Ayusawa, N Sumitomo, T Okada, K Harada
}

Heart 2002;87:559-565

See end of article for authors' affiliations ......................

Correspondence to: Dr Nobutaka Noto Department of Paediatrics and Cardiology, Nihon University School of Medicine, 30-1 Oyaguchi, Itabashi-ku, Tokyo

173-8610, Japan: nnoto@med.email.ne.jp

Accepted

16 January 2002

\begin{abstract}
Objective: To investigate whether transthoracic Doppler echocardiography (TTE) can reliably measure the coronary flow reserve in the left anterior descending coronary artery in children with Kawasaki disease.

Design: Coronary flow velocity in the distal left anterior descending coronary artery was measured by TTE and was compared with that obtained by intracoronary Doppler guide wire. The ratio of maximum hyperaemia (intravenous administration of adenosine triphosphate, $160 \mu \mathrm{g} / \mathrm{kg} / \mathrm{min}$ ) to baseline peak (mean) diastolic coronary flow velocity in the distal artery was used as an estimate of coronary flow reserve.

Setting: University hospital.

Patients: 10 patients with significant left anterior descending coronary stenosis $1>70 \%$ diameter stenosis) (group A) in the proximal or middle portion of the artery and 14 patients (group B) without significant stenosis, all with Kawasaki disease documented by previous coronary angiography.

Results: The reduced hyperaemic coronary flow velocity in group A compared with group B resulted in a markedly lower coronary flow reserve, derived from both peak diastolic velocity and mean diastolic velocity by either technique of investigation. Multivariate analysis identified the best predictor of left anterior descending coronary artery stenosis to be a coronary flow reserve of $\leqslant 2.2$, derived from mean diastolic flow velocity measured using TTE (sensitivity $90 \%$, specificity 100\%, accuracy $96 \%$ ). A good correlation was found between diastolic velocity derived values for coronary flow reserve measured using both TTE and Doppler guide wire $(r=0.92, p=0.0001)$.

Conclusions: Coronary flow reserve in the distal left anterior descending coronary artery can be accurately measured using TTE without any intravascular instrumentation in children with Kawasaki disease.
\end{abstract}

$\mathrm{F}$ unctional impairment of a coronary artery can be assessed by measuring coronary flow reserve-that is, the capacity of the artery to allow increased blood flow when needed. The concept of coronary flow reserve is receiving increased attention because of its clinical and physiological importance. ${ }^{1}$ Measurements of coronary flow velocity and flow reserve during cardiac catheterisation were first done by Wilson et al in 1985, ${ }^{2}$ and since then intracoronary Doppler guide wire recording has been shown to be a highly accurate way of measuring coronary flow velocity, and has been used for evaluating various clinical problems. ${ }^{34}$

Recent studies have suggested that blood flow velocity in the proximal left anterior descending coronary artery can be determined by transoesophageal Doppler echocardiography. ${ }^{5-7}$ Furthermore, new developments in ultrasound technology now enable low velocity flow signals in the epicardial coronary arteries to be detected by colour and pulsed Doppler echocardiography from transthoracic windows, though the value of these procedures has not yet been established in any large series of cases. ${ }^{8-10}$

If measurements of intracoronary flow velocity by transthoracic echocardiography (TTE) could be shown to be equivalent to those obtained by Doppler guide wire, then TTE could be used in situations where intravascular instrumentation is unnecessary or undesirable.

Our aim in this study was to investigate whether TTE can be used to measure coronary flow reserve reliably in the distal portion of the left anterior descending coronary artery in comparison with measurements obtained by Doppler guide wire in children with Kawasaki disease.

\section{METHODS \\ Patients}

The study population consisted of 25 consecutive patients ( 15 boys and 10 girls; age range 4 to 16 years, mean (SD) age 9.0 (3.6) years) who were undergoing routine coronary angiography for the assessment of coronary sequelae of Kawasaki disease. All patients had isolated left anterior descending coronary artery disease, with coronary ectasia or aneurysms in either the proximal or the middle portion of the artery in the acute or convalescent phase, documented by previous cross sectional echocardiography or coronary angiography. The interval from the initial onset of illness ranged from two to nine years (mean 5.8 (2.0) years)

Of the 25 patients, 10 had a significant stenotic lesion in the proximal or middle portion of the left anterior descending coronary artery (group A; > 70\% diameter stenosis; mean age 9.3 (4.0) years). The remainder did not have a significant stenotic lesion in this vessel (group B; mean age 8.9 (3.6) years). There were no coronary artery lesions apart from those in the left anterior descending coronary artery in either group.

Thirteen patients were receiving concurrent medical treatment. Eight were on aspirin only, while five were receiving both aspirin and warfarin to prevent coronary thrombosis. None of the patients was receiving a $\beta$ blocker, a calcium channel blocker, or nitrates. All patients were free of symptoms during their normal daily activities. None had a clinical history or ECG signs of previous myocardial infarction. Ventricular function was normal and there was no echocardiographic evidence of left ventricular hypertrophy, valvar heart disease, or left ventricular wall motion abnormality at rest.

The study was approved by the human research ethics committee of our institution, and written informed consent for 

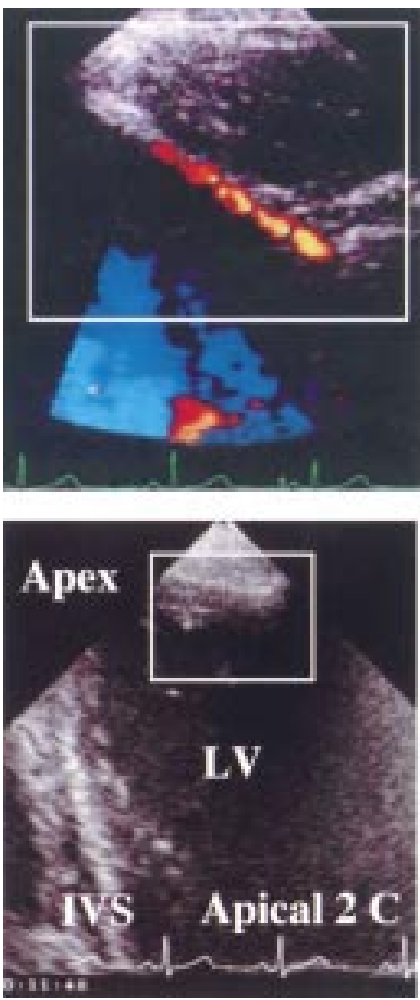
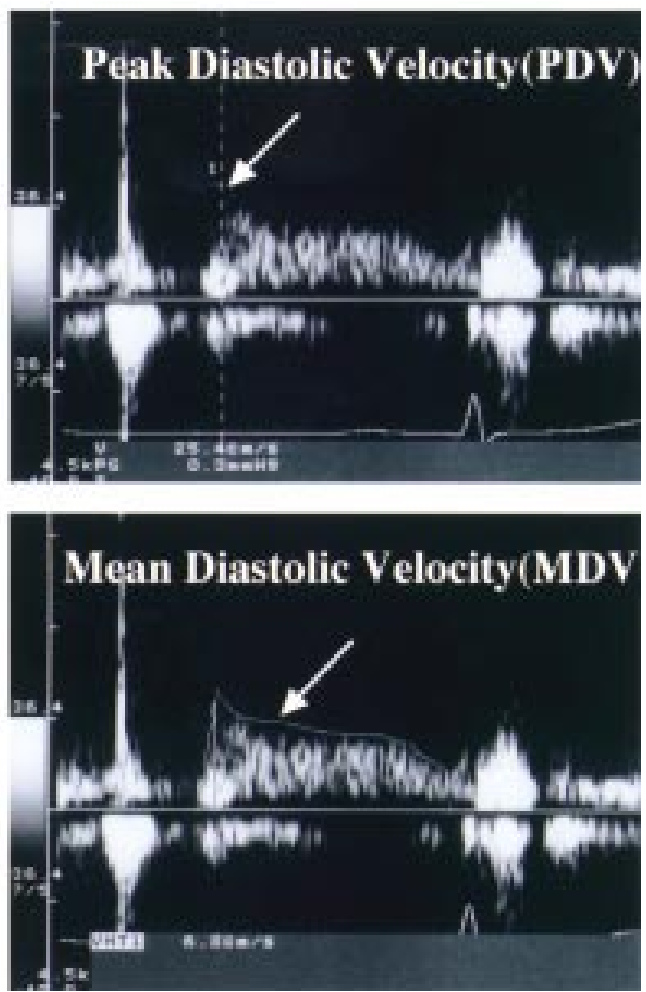

Figure 1 Lower left panel: detection of the coronary blood flow signals in the distal left anterior descending coronary artery (LAD) from the apical two chamber view. Coronary blood flow signals in the distal LAD were sought under the guidance of colour flow mapping at the apical portion of the left ventricle (top left panel). The pulsed wave sampling volumes were placed over the these signals, and Doppler spectral flow recordings of the peak diastolic velocity (top right panel) and the mean diastolic velocity (bottom right panel) were made. IVS, interventricular septum; LV, left ventricle; 2C, two chamber.

participation was obtained from all parents or legal guardians of the patients.

\section{Echocardiographic equipment}

Heart rate, ECG, and frequent cuffed blood pressure measurements (Dynamap, Critikon Inc, Tampa, Florida, USA) were monitored continuously throughout the study. TTE was done using a Toshiba SSA-380A diagnostic system and a 7.5 MHz transducer (Toshiba, Tokyo, Japan), with the patient in the left lateral decubitus position. To obtain optimal colour Doppler flow signals in the epicardial coronary arteries, we paid special attention to the colour Doppler flow settings, using a lowered velocity range ( 16.1 or $17.4 \mathrm{~cm} / \mathrm{s})$, a lowered cut off frequency for the Doppler filter ( 400 to $440 \mathrm{~Hz}$ ) to eliminate the clutter signal from the myocardium, a high pulse repetition frequency ( 6.0 to $6.5 \mathrm{kHz}$ ), and the narrowest scan range possible for a high frame rate $(\geqslant 25 \mathrm{~Hz}$ ) during high frequency tissue imaging (7.5 MHz).

\section{Doppler echocardiographic measurements}

The transducer was manipulated to visualise the apical portion of the left ventricle from the apical two chamber view, and then rotated by a small amount to obtain the optimum Doppler flow signals in the distal portion of left anterior descending coronary artery, under guidance of Doppler colour flow mapping. Measurement of Doppler spectral flow velocity in the distal left anterior descending artery has been described in detail by Hozumi et al and Caiati et al. ${ }^{90}$ Doppler signals from the epicardial coronary artery were readily recognised, with their small systolic and large diastolic components. In most instances, we were unable to display the complete systolic Doppler spectral envelop during hyperaemia because of excessive cardiac motion. For this reason we confined our analysis of epicardial coronary blood flow to the diastolic Doppler spectral envelop.
Digitised Doppler spectral envelops for peak diastolic velocity and mean diastolic velocity were averaged from three cardiac cycles to compute the coronary flow velocity indices (fig 1). All signals were recorded continuously on s-VHS videotape, and portions were also captured in cineloop format and stored digitally to facilitate off-line measurements.

\section{Coronary angiography}

Coronary angiography was done on all patients by the femoral approach within 24 hours of TTE, using standard catheters and conventional views. The angiograms were analysed by two experienced angiographers with no knowledge of the Doppler results. The degree of stenosis was measured in comparison with adjacent, normal appearing vessel segments using digital electronic calipers (Digitizer KD 4300, Graphtec Co, Tokyo, Japan) in two orthogonal views. ${ }^{112}$ The intracoronary collateral circulation was classified by the Rentrop grading (ranging from grade $0=$ no visible filling of any collateral channels to grade $3=$ complete filling of the vessel being dilated). ${ }^{13}$ Calibration to diagnostic catheter dimensions was used to correct for differences in magnification.

\section{Intracoronary Doppler guide wire measurements}

After routine coronary angiography, a Doppler guide wire (Flowire, Cardiometrics Inc, Mountain View, California, USA) was advanced through a $5 \mathrm{~F}$ guiding Judkins-type coronary catheter to a position 2 to $3 \mathrm{~cm}$ distal to the stenotic lesion, to avoid placement in a side branch or in the poststenotic velocity jet. The Doppler guide wire was a 0.014 inch $(0.36 \mathrm{~mm})$ diameter, $175 \mathrm{~cm}$ long, flexible, steerable guide wire with a 12 $\mathrm{MHz}$ ultrasound transducer integrated into the tip, and was connected to a Cardiometrics Flowmap instrument. Spectral Doppler flow velocity was measured as in previous studies. ${ }^{34}$ The peak diastolic velocity, which represents maximum peak velocity, and the mean diastolic velocity, which represents the 
Table 1 Coronary flow velocity dynamics in patients with or without significant stenosis of the left anterior descending coronary artery

\begin{tabular}{|c|c|c|c|c|c|c|c|c|c|c|c|c|}
\hline & \multicolumn{4}{|l|}{ TTE } & \multicolumn{4}{|l|}{ DGW } & \multicolumn{2}{|l|}{ TTE } & \multicolumn{2}{|l|}{ DGW } \\
\hline & $\begin{array}{l}\text { PDV-b } \\
(\mathrm{cm} / \mathrm{s})\end{array}$ & $\begin{array}{l}\text { PDV-h } \\
(\mathrm{cm} / \mathrm{s})\end{array}$ & $\begin{array}{l}\text { MDV-b } \\
(\mathrm{cm} / \mathrm{s})\end{array}$ & $\begin{array}{l}\text { MDV-h } \\
(\mathrm{cm} / \mathrm{s})\end{array}$ & $\begin{array}{l}\text { PDV-b } \\
(\mathrm{cm} / \mathrm{s})\end{array}$ & $\begin{array}{l}\text { PDV-h } \\
(\mathrm{cm} / \mathrm{s})\end{array}$ & $\begin{array}{l}\text { MDV-b } \\
(\mathrm{cm} / \mathrm{s})\end{array}$ & $\begin{array}{l}\text { MDV-h } \\
(\mathrm{cm} / \mathrm{s})\end{array}$ & $\begin{array}{l}\text { CFR } \\
\text { (PDV) }\end{array}$ & $\begin{array}{l}\text { CFR } \\
\text { (MDV) }\end{array}$ & $\begin{array}{l}\text { CFR } \\
\text { (PDV) }\end{array}$ & $\begin{array}{l}\text { CFR } \\
\text { (MDV) }\end{array}$ \\
\hline \multicolumn{13}{|c|}{ Group A (LAD stenosis $>70 \%$ ) } \\
\hline Mean & 35.2 & 68.1 & 16.5 & 31.7 & 41.2 & 77.8 & 26.3 & 50.7 & 1.9 & 1.9 & 1.9 & 1.9 \\
\hline SD & 12.4 & 25.6 & 6.6 & 12.7 & 13.3 & 25.9 & 7.6 & 17.8 & 0.2 & 0.3 & 0.3 & 0.2 \\
\hline \multicolumn{13}{|c|}{ Group B (LAD stenosis $\leqslant 70 \%$ ) } \\
\hline Mean & 30.8 & 83.7 & 13.3 & 36.6 & 35.1 & 99.2 & 21.9 & 67.7 & $2.8^{*}$ & $2.8^{*}$ & $3.0^{*}$ & $3.1^{*}$ \\
\hline SD & 7.5 & 16.6 & 5.6 & 13.4 & 8.9 & 25.3 & 6.9 & 16.9 & 0.4 & 0.4 & 0.5 & 0.5 \\
\hline
\end{tabular}

${ }^{*} p<0.0001 \vee$ group $A$.

b, base; CFR, coronary flow reserve; DGW, Doppler guide wire; h, hyperaemia; LAD, left anterior descending coronary artery; MDV, mean diastolic velocity; PDV, peak diastolic velocity; TTE, transthoracic echocardiography.

average diastolic peak velocity in the Doppler guide wire measurements, were obtained from the distal portion of the artery, and four consecutive beats showing a satisfactory envelope were averaged.

\section{Calculation of coronary flow reserve}

After baseline measurements of coronary flow velocity, heart rate, and blood pressure had been obtained, adenosine triphosphate (ATP) was infused into a right antecubital vein at a dose of $160 \mu \mathrm{g} / \mathrm{kg} / \mathrm{min}$ for six minutes through a high flow infusion pump, and data were recorded at baseline and during hyperaemia (the last minute of ATP infusion). The ratio of the hyperaemic peak diastolic velocity (or mean diastolic velocity) to the baseline peak diastolic velocity (or mean diastolic velocity) was calculated as an index of coronary flow reserve in the distal left anterior descending coronary artery.

\section{Interobserver and intraobserver variability}

To evaluate the effect of observer variability on the measurement of coronary flow velocity by TTE, two independent observer (NN, KK) analysed the measurements of coronary flow velocity in the same patient in the same state (either baseline or during hyperaemia). Intraobserver variability was also analysed using pairs of measurements made by the same observer $(\mathrm{NN})$ on the coronary flow velocity in the same patient at least two weeks apart.

\section{Statistical analysis}

Data are presented as mean (SD). The differences between the two groups for the parametric data were tested using an unpaired two tailed $t$ test. Differences between baseline and hyperaemic datasets within the same groups were tested using a paired two tailed $t$ test as appropriate. The coronary flow velocity and coronary flow reserve calculated by TTE were compared with values obtained by Doppler guide wire using linear regression analysis. Linear stepwise regression analysis was used to determine the best predictors of left anterior descending coronary artery stenosis. The sensitivity and specificity of coronary flow reserve as a predictor of significant coronary artery stenosis were measured in the traditional manner. Agreement between the two measurements was tested by the method of Bland and Altman. ${ }^{14}$ A probability (p) value of $<0.05$ was considered to indicate statistical significance.

\section{RESULTS}

Twenty four of the 25 patients had technically adequate Doppler measurements for analysis. We were not able to obtain good quality Doppler signals from the distal left anterior descending coronary artery in one patient, in whom the vessel could not be visualised during colour flow mapping by TTE in our initial study.

\section{Coronary angiographic findings}

Five coronary aneurysms were located in the proximal of the left anterior descending coronary artery and 14 in the middle portion of the artery. Seventeen stenotic lesions were located at either the entry or the exit of each aneurysm. The mean per cent diameter stenosis of each group was $79(6) \%$ in group A and $17(20) \%$ in group B, respectively. Significant collateral circulation (Rentrop grade $\geqslant 2$ ) was seen in one patient with $90 \%$ stenosis in the proximal portion of the artery.

\section{Haemodynamic variables}

In group A, there was no significant change between baseline and hyperaemia in heart rate, systolic blood pressure, mean blood pressure, or rate-pressure product obtained by both TTE and Doppler guide wire. In group B, there was an increase in heart rate during hyperaemia compared with baseline: 95.3 (13.2) $v 85.5$ (8.2) beats/min with TTE; 90.9 (11.8) $v 80.5$ (10.9) beats/min with Doppler guide wire; $\mathrm{p}<0.05$. However, we found no differences in the other measured variables in group B. Hence there was no significant difference between the values of rate-pressure product $\left(\times 10^{3}\right)$ obtained by either TTE or Doppler guide wire at baseline $(8.6(1.1) v 8.4(1.5)$ in group A; $8.3(0.9) v 8.4(0.9)$ in group B, respectively) or during hyperaemia $(9.3$ (1.2) $v 8.8$ (1.5) in group A; 9.1 (1.4) $v 8.7$ (1.0) in group B).

Most patients tolerated the ATP infusion well, but three had side effects (flushing in one, chest discomfort in one, and nausea in one). These side effects were self limiting and resolved spontaneously with discontinuation of the infusion. No patient developed atrioventricular block, premature atrial contraction, or premature ventricular contraction during the ATP infusion.

\section{Velocimetry}

The results of the Doppler echocardiographic and Doppler guide wire measurements are given in table 1 . At baseline, the coronary flow velocity values in the distal artery were slightly higher in group A than in group B, but the difference did not reach statistical significance with either TTE or Doppler guide wire. During hyperaemia, the coronary flow velocity values in the distal artery were increased significantly compared with baseline in both groups and using both types of measurement (all $p<0.001$ ). The values of hyperaemic coronary flow velocity tended to increase more in group B than in group A. The reduced hyperaemic flow velocity in group A resulted in markedly reduced values for the following: coronary flow reserve derived from peak diastolic velocity by TTE $(1.9(0.2) v$ $2.8(0.4), \mathrm{p}<0.0001)$; mean diastolic velocity by TTE (1.9 $(0.3) v 2.8(0.4), \mathrm{p}<0.0001)$; peak diastolic velocity by Doppler guide wire (1.9 (0.3) v $3.0(0.5), \mathrm{p}<0.0001)$; and mean diastolic velocity by Doppler guide wire $(1.9(0.2) \vee 3.1$ (0.5), p < 0.0001) (figs 2 and 3). 


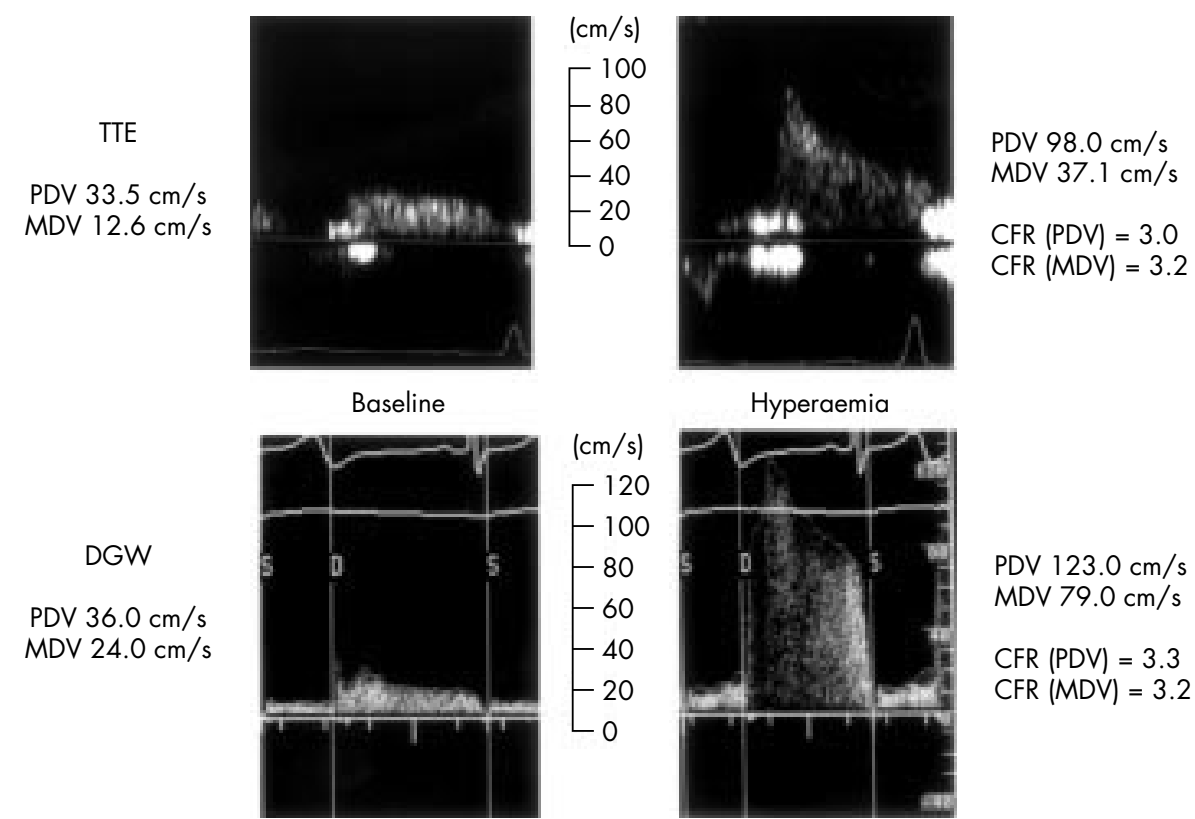

Figure 2 Spectral Doppler flow recordings of the distal left anterior descending coronary artery (LAD) in a patient without significant LAD stenosis, measured using transthoracic Doppler echocardiography (TTE) (top panels) and Doppler guide wire (DGW) (bottom panels). MDV, mean diastolic velocity; PDV, peak diastolic velocity.

By discriminant analysis, the best cut off values for predicting a significant left anterior descending coronary artery stenosis were:

- peak diastolic velocity of $80 \mathrm{~cm} / \mathrm{s}$ during hyperaemia by Doppler guide wire (sensitivity 60\%, specificity 86\%, $\mathrm{p}=0.0283$ )

- mean diastolic velocity of $60 \mathrm{~cm} / \mathrm{s}$ during hyperaemia by Doppler guide wire (sensitivity $80 \%$, specificity $85 \%$, $\mathrm{p}<0.0069$ )

- coronary flow reserve of 2.2 derived from peak diastolic velocity by Doppler guide wire (sensitivity $80 \%$, specificity $100 \%, \mathrm{p}<0.0001)$;
- coronary flow reserve of 2.2 derived from mean diastolic velocity by Doppler guide wire (sensitivity $80 \%$, specificity $92 \%, \mathrm{p}<0.0001)$;

- coronary flow reserve of 2.2 derived from peak diastolic velocity by TTE (sensitivity 90\%, specificity 93\%, $\mathrm{p}<0.0001)$;

- coronary flow reserve of 2.2 derived from mean diastolic velocity by TTE (sensitivity 90\%, specificity 100\%, $\mathrm{p}<0.0001$ ) (table 2).

Using stepwise multiple linear regression, the best predictor of coronary artery stenosis was coronary flow reserve derived from mean diastolic velocity by TTE. A good correlation was

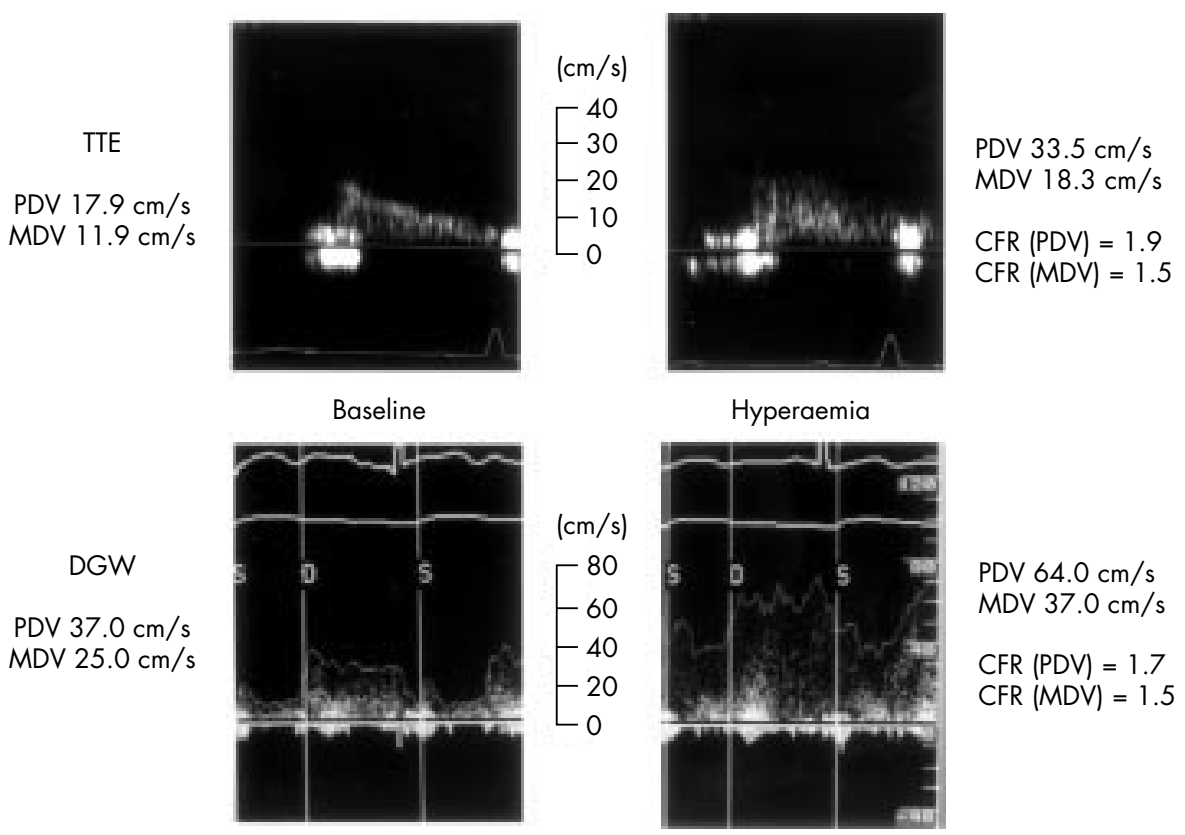

Figure 3 Spectral Doppler flow recordings of the distal left anterior descending coronary artery (LAD) in a patient with $90 \%$ stenosis in the middle portion of LAD measured using transthoracic Doppler echocardiography (TTE) (top panels) and Doppler guide wire (DGW) (bottom panels). MDV, mean diastolic velocity; PDV, peak diastolic velocity. 
Table 2 Results of discriminant analysis

\begin{tabular}{llllll}
\hline Variable & Sensitivity (\%) & Specificity (\%) & Accuracy (\%) & Cut off value & p Value \\
\hline CFRTTE(MDV) & 90 & 100 & 96 & 2.2 & $<0.0001$ \\
CFRTTE(PDV) & 90 & 93 & 92 & 2.2 & $<0.0001$ \\
CFRDGW(MDV) & 80 & 100 & 92 & 2.2 & $<0.0001$ \\
CFRDGW(PDV) & 80 & 100 & 92 & 2.2 & $<0.0001$ \\
MDVDGW-h (cm/s) & 80 & 85 & 79 & 60 & 0.0069 \\
PDVDGW-h (cm/s) & 60 & 86 & 75 & 80 & 0.0283 \\
\hline CFRDGW(MDV), coronary flow reserve derived from mean diastolic velocity by intracoronary Doppler \\
guidewire; CFRDGW(PDV), coronary flow reserve derived from peak diastolic velocity by intracoronary \\
Doppler guidewire; CFRTTE(MDV), coronary flow reserve derived from mean diastolic velocity by \\
transthoracic echocardiography; CFRTTE(PDV), coronary flow reserve derived from peak diastolic velocity by \\
transthoracic echocardiography; h, hyperaemia; MDVDGW-h, mean diastolic velocity measured by \\
intracoronary Doppler guidewire during hyperaemia; PDVDGW-h, peak diastolic velocity measured by \\
intracoronary Doppler guidewire during hyperaemia.
\end{tabular}

found between the values for coronary flow reserve derived from mean diastolic velocity by TTE and by Doppler guide wire (slope $=1.15, \mathrm{SEE}=0.106, r=0.92, \mathrm{p}=0.0001)$ (fig 4, left panel). A plot of the difference between the values for coronary flow reserve derived from mean diastolic velocity by TTE and by Doppler guide wire versus the averaged value of these two measurements showed good agreement (fig 4, right panel).

\section{Variability}

The interobserver variability was low. Altogether 22 pairs of measurements were made in seven patients. There was a high correlation between the measurements of coronary flow velocity made by the two observers $(r=0.93, \mathrm{p}=0.0001$, SEE $=0.04 \mathrm{~cm} / \mathrm{s})$. The intraobserver variability, which was assessed from 18 pairs of measurements in eight patients, was also low $(r=0.95, \mathrm{p}=0.0001, \mathrm{SEE}=0.03 \mathrm{~cm} / \mathrm{s})$.

\section{DISCUSSION}

In this study, we were able to measure coronary flow reserve non-invasively using TTE in patients with Kawasaki disease. We found a good correlation between these measurements and ones made using a Doppler guide wire in this selected group of patients.

\section{Impact of the assessment of coronary flow reserve in Kawasaki disease}

Kawasaki disease is an acute inflammatory process affecting the arterial wall which results in panvasculitis in infants and young children. Coronary artery aneurysms develop in about $10 \%$ of children with this disease and may lead to myocardial ischaemia. ${ }^{15}$ The coronary artery lesions that develop as complications of the disease are important in determining the prognosis. Recent studies using single photon emission computed tomography (SPECT) have shown that there are exercise induced regional perfusion abnormalities in some Kawasaki patients with normal epicardial coronary arteries. ${ }^{16}$ The cause of this impairment of hyperaemic blood flow is unknown, though it may involve microvascular lesions like those reported in diabetes or cardiomyopathy; these cannot be visualised by coronary angiography or conventional cross sectional echocardiography. ${ }^{17}$ It is apparent from these findings that the assessment of coronary artery lesions in Kawasaki disease should not only be based on coronary angiographic findings but also on tests of coronary function.

\section{Comparison with previous studies}

Investigating coronary flow reserve in adult patients with transoesophageal echocardiography, Iliceto et al showed significant differences in values derived from peak and mean diastolic velocity ratio between patients with left anterior descending coronary artery stenosis and normal subjects (coronary flow reserve peak, $1.46(0.45) \quad v \quad 3.22(0.96)$; coronary flow reserve mean, $1.48(0.49) v 3.04(0.88)){ }^{6}$ Similar results were reported by Redberg et al and Hutchison et al using transoesophageal echocardiography. ${ }^{78}$ In our study, the TTE values for coronary flow reserve in patients with
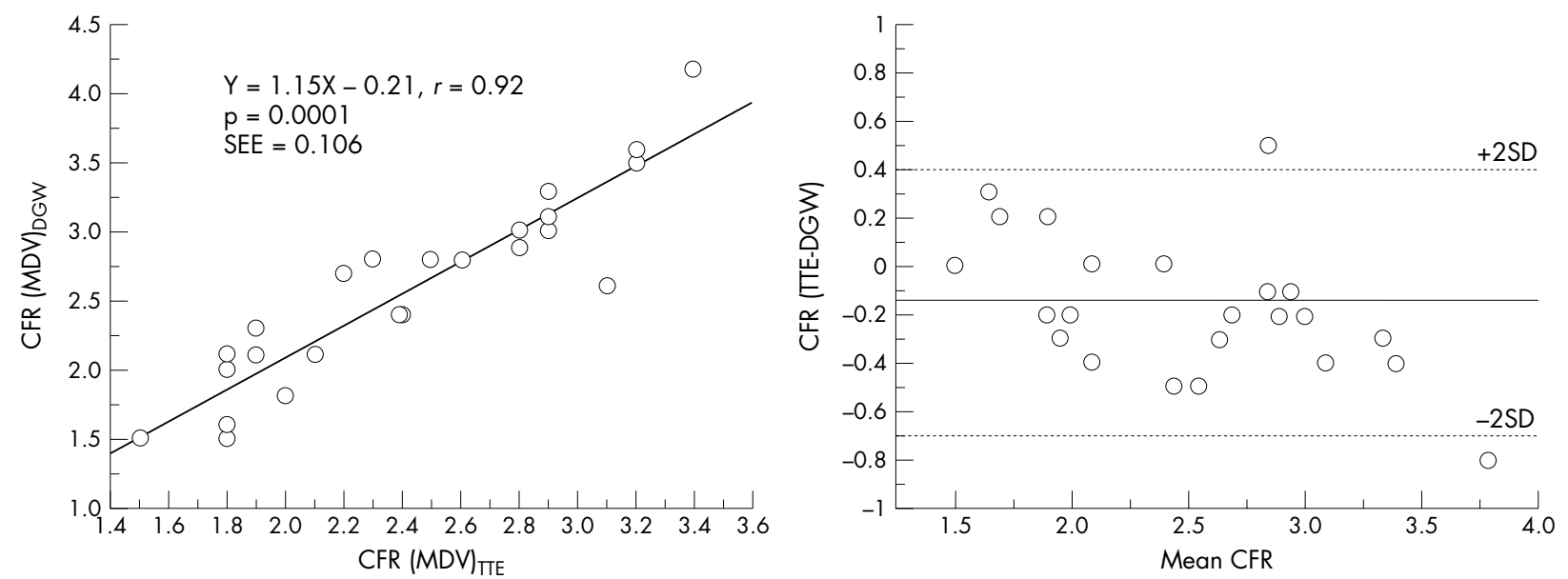

Figure 4 Left panel: Relation between the values of coronary flow reserve (CFR) derived from mean diastolic velocity (MDV) obtained using transthoracic Doppler echocardiography (TTE) and Doppler guide wire (DGW). The regression equation with the correlation coefficient is shown at the top. Right panel: Plot of the differences between the values of CFR derived from MDV measured using TTE and DGW $v$ the average of the two values. The dotted lines show the boundaries of mean and 2 SD. CFR(MDV) $)_{\text {DGw }}$ coronary flow reserve derived from mean diastolic velocity measured using Doppler guide wire; CFR(TTE-DGW), the difference between the values of CFR derived from MDV by transthoracic Doppler echocardiography and Doppler guide wire. 
significant left anterior descending coronary artery stenosis (coronary flow reserve peak, $1.9(0.20)$; coronary flow reserve mean, $1.9(0.30))$ were somewhat higher than those in previous studies. This probably reflects the different patient population and the degree of stenosis. Our subjects all had $>70 \%$ stenosis, and eight of the 10 patients in this group had $>80 \%$ stenosis. Other factors that could be involved in differences between our study and other reports include the degree of collateral circulation and differences in stenosis morphology in coronary lesions in children with Kawasaki disease and adult patients with atherosclerosis. In contrast, the coronary flow reserve values in our control group (peak, $2.8(0.4)$; mean, $2.8(0.4)$ ) were slightly lower than those reported by Ileceto et al and Hutchison et al. This again reflects differences in definitions of normal populations. Our control group contained some patients with coronary artery stenoses, but all less than $50 \%$, while in the studies by Ileceto and Hutchison the control groups included only patients with no stenotic lesions. Additional imaging by intravascular ultrasound at the time of coronary angiography may yield interesting new information on the pathophysiology of the coronary circulation in children with Kawasaki disease.

\section{Coronary flow reserve in children with Kawasaki disease}

Little is known about coronary flow reserve in Kawasaki disease, with or without coronary artery stenosis. Recently, Hamaoka et al reported a significant age related increase in coronary flow reserve in all three coronary arteries in Kawasaki patients, but normal coronary angiography by using Doppler guide wire on intracoronary ATP administration, and no significant changes in haemodynamic variables. ${ }^{19}$ From their studies, the normal value for coronary flow reserve in the left anterior descending coronary artery was $2.07(0.30)$ for the age group one to five years, $2.69(0.40)$ for the age group six to 15 years, and $3.50(0.45)$ for the age group 16 to 23 years. Their value for the age group six to 15 years is consistent with our earlier control value (mean age 8.9 years) using TTE. ${ }^{8}$ The cause of the lower flow reserve values in younger children has not been determined, but developmental immaturity of the coronary arteries is a possibility. It is also likely that other factors, such as sympathetic stimuli and changes in heart rate, can influence coronary flow velocity and flow reserve in children without significant coronary artery stenosis. In fact, three of the five patients with a mean coronary flow reserve of less than 2.6 measured by TTE in our group B were aged under six years, while the remaining two patients tended to have higher heart rates at baseline than the remainder of group B-and therefore a higher baseline coronary flow velocity resulting in lower coronary flow reserve values. Nonetheless, a coronary flow reserve of $>2.2$ obtained using TTE is useful in excluding significant left anterior descending coronary artery stenosis in patients under 16 years of age with Kawasaki disease.

\section{Limitations of the study}

There are several possible limitations of our study. First, as we could not perform the Doppler guide wire and TTE recordings simultaneously, the haemodynamic conditions in the two studies may not have been identical. Moreover, it is possible that the sampling sites in the artery may have been different with the two techniques. These factors could account for some of the differences between coronary flow velocity values measured by Doppler guide wire and by TTE. Second, we used the diastolic flow velocity for measurements of coronary flow reserve without taking systolic flow velocity into account. However, another recent study has shown that augmentation of coronary flow velocity by intra-aortic balloon pumping, assessed using peak diastolic velocity, was similar to that assessed using the flow velocity integral of the entire left anterior descending coronary artery flow velocity signal. ${ }^{20}$
Moreover, it is difficult to differentiate the small Doppler spectral envelop of systole from clutter signals when assessing the Doppler waveform using on-line fast Fourier transformation. Thus for measuring coronary flow velocity in the distal left anterior descending coronary artery, we believe that it is satisfactory to make the calculations employing only the diastolic flow signals.

\section{Clinical implications}

In the absence of significant haemodynamic changes, serial coronary flow reserve measurements are highly reproducible in humans. ${ }^{21}$ Thus sequential determinations of coronary flow reserve obtained with TTE may be a valuable clinical tool in the longitudinal assessment of patients with coronary artery lesions. Potential advantages of TTE include the fact that it is non-invasive, widely available, relatively inexpensive, and does not require any intravascular instrumentation during measurement of coronary flow velocity. Thus it can even be done in an ambulatory setting. From a clinical standpoint, knowledge of coronary flow reserve in patients with Kawaski disease and coronary involvement would be clinically useful in planning measures to prevent myocardial ischaemia or sudden cardiac death. It might also be useful in other entities such as graft atherosclerosis or chronic rejection following heart transplantation. Further clinical trials of this promising approach are warranted, including measurement of coronary flow velocity in the other coronary arteries from different imaging windows.

\section{Conclusions}

Values of coronary flow reserve in the distal left anterior descending coronary artery assessed by transthoracic echocardiography correlated closely with those assessed by Doppler guide wire. Our data therefore indicate that coronary flow reserve can be measured accurately using TTE without any intravascular instrumentation in this selected cohort. Further studies in larger groups of unselected patients and on other coronary vessels are necessary to assess the advantages and clinical limitations of this method for evaluating the functional significance of coronary artery lesions in children with Kawasaki disease.

\section{ACKNOWLEDGEMENTS}

This work was supported in part by a grant for Kawasaki disease from the Ministry of Health and Welfare of Japan.

.....................

Authors' affiliations

N Noto, K Karasawa, H Kanamaru, M Ayusawa, N Sumitomo, T Okada, K Harada, Department of Paediatrics and Cardiology, Nihon University School of Medicine, Tokyo, Japan

\section{REFERENCES}

1 Hoffman JIE. Maximal coronary flow and the concept of coronary vascular reserve. Circulation 1984;70:153-8.

2 Wilson RF, Laughlin DE, Ackell PH, et al. Transluminal, subselective measurement of coronary artery blood flow velocity and vasodilator reserve in man. Circulation 1985:72:82-92.

3 Doucette JW, Corl PO, Payne HM, et al. Validation of a Doppler guide wire for intravascular measurement of coronary artery flow velocity. Circulation 1992;85:1899-911.

4 Ofilli EO, Kern M, Lavovitz AJ, et al. Analysis of coronary blood flow velocity dynamics in angiographically normal and stenosed arteries before and after endolumen enlargement by angioplasty. J Am Coll Cardiol 1993;21:308-16.

5 Yoshida K, Yoshikawa J, Hozumi T, et al. Detection of left main coronary artery stenosis by transesophageal color Doppler and two-dimensional echocardiography. Circulation 1990;81:1271-6.

6 Iliceto S, Marangelli V, Memmola C, et al. Transesophageal Doppler echocardiography evaluation of coronary blood flow velocity in baseline conditions and during dipyridamole-induced coronary vasodilation. Circulation 1991:83:61-9.

7 Redberg RF, Sobol Y, Chou TM, et al. Adenosine-induced coronary vasodilatation during transesophageal Doppler echocardiography. Rapid 
and safe measurement of coronary flow reserve ratio can predict significant left anterior descending coronary stenosis. Circulation 1995;92:190-6.

8 Noto N, Karasawa K, Ayusawa M, et al. Measurement of coronary flow reserve in children by transthoracic Doppler echocardiograph. Am J Cardiol 1997:80:1638-9.

9 Hozumi T, Yoshida K, Ogata Y, et al. Noninvasive assessment of significant left anterior descending coronary artery stenosis by coronary flow velocity reserve with transthoracic color Doppler echocardiography. Circulation 1998:97:1557-62.

10 Caiati C, Montaldo C, Zedda N, et al. A new non-invasive method for coronary flow reserve assessment: contrast-enhanced transthoracic second harmonic echo Doppler. Circulation 1999;99:771-8.

11 Nakanishi T, Takao A, Nakazawa M, et al. Mucocutaneous lymph node syndrome: clinical, hemodynamic and angiographic features of coronary obstructive disease. Am J Cardiol 1985;55:662-8.

12 Kawasaki Disease Research Committee. Diagnostic criteria of the cardiac involvement in Kawasaki disease. Tokyo, Japan: Ministry of Health and Welfare, 1983.

13 Cohen M, Rentrop KP. Limitation of myocardial ischemia by collateral circulation during sudden controlled coronary artery occlusion in human subjects: a prospective study. Circulation 1986;74:469-76.

14 Bland JM, Altman DG. Statistical methods for assessing agreement between two methods of clinical measurement Lancet 1986:i-307-10.
15 Kato H, Inove O, Akagi T. Kawasaki disease: cardiac problem and management. Pediatr Rev 1988;9:209-17.

16 Paridon S, Galioto F, Vincent J, et al. Exercise capacity and incidence of myocardial perfusion defects after Kawasaki disease in children and adolescents. J Am Coll Cardiol 1995;25: 1420-4.

17 Fujiwara T, Fujiwara $\mathrm{H}$, Nakano H. Pathological features of coronary arteries in children with Kawasaki disease in which coronary arterial aneurysm was absent at autopsy. Circulation 1988;78:345-50.

18 Hutchison SJ, Soldo SJ, Gadallah S, et al. Determination of coronary flow measurements by transesophageal echocardiography: dependence of flow velocity reserve on the location of stenosis. Am Heart $J$ 1997; 133:44-52.

19 Hamaoka K, Onouchi Z, Ohmochi Y, et al. Evaluation of coronary flow dynamics and flow reserve in patients with Kawasaki disease by means of a Doppler guide wire. J Am Coll Cardiol 1998;31:833-40.

20 Hutchison SJ, Thaker K, Chandraratna PA. Effect of intraaortic counterpulsation on flow velocity in stenotic left main coronary arteries using transesophageal echocardiography. Am J Cardiol 1994; 15:1063-5.

21 McGinn AL, White CW, Wilson RF. Interstudy variability of coronary flow reserve: influence of heart rate, arterial blood pressure and ventricular preload. Circulation 1990;81:1319-30.

\section{IMAGES IN CARDIOLOGY}

\section{Biventricular hypertrophic cardiomyopathy with lone obstruction to right ventricular oufflow}

A

45 year old man with a history of hypertrophic obstructive cardiomyopathy (HOCM) was admitted to our hospital because of a syncope. The diagnosis of HOCM had initially been made at cardiac catheterisation in 1975 . He had been given $120 \mathrm{mg}$ propranolol daily and his subsequent clinical history was uneventful. Since 1981 he had been undergoing echocardiography every two years. Echocardiography revealed notably hypertrophied ventricles and severe obstruction to the left ventricular outflow (in 1985 continuous wave Doppler was performed disclosing a peak gradient of $80 \mathrm{~mm} \mathrm{Hg}$ ). The gradient to the left ventricular outflow decreased over the years and in 1998 no gradient to both left and right ventricular outflow was detected. In March 2000 he had paroxysmal atrial flutter with syncope; sinus rhythm was restored after intravenous flecainide. Cross sectional echocardiography showed pronounced asymmetric left ventricular hypertrophy and thickened right ventricular muscular components (prominently the crista supraventricularis). Impediment to right ventricular outflow was caused by simultaneous systolic contraction of the hypertrophied ventricular septum and crista supraventricularis (top right: LV, left ventricle; RVOT, right ventricular outflow tract). Colour Doppler demonstrated turbulent flow across the right ventricular outflow tract and laminar flow across the left ventricular outflow (bottom right: LA, left atrium; LVOT, left ventricular outflow tract). Right ventricular outflow peak gradient was $45 \mathrm{~mm} \mathrm{Hg}$. Valsalva manoeuvre did not induce any variation in both right and left ventricular outflow tract Doppler velocities. Cardiac catheterisation confirmed the echo findings and showed an intramural left anterior descending coronary artery.

Right ventricular outflow tract pressure gradients may occur infrequently in HOCM and are usually associated with subaortic gradients. To our knowledge this is the first case in which the obstruction switched from the left to the right ventricular outflow in a long term follow up.
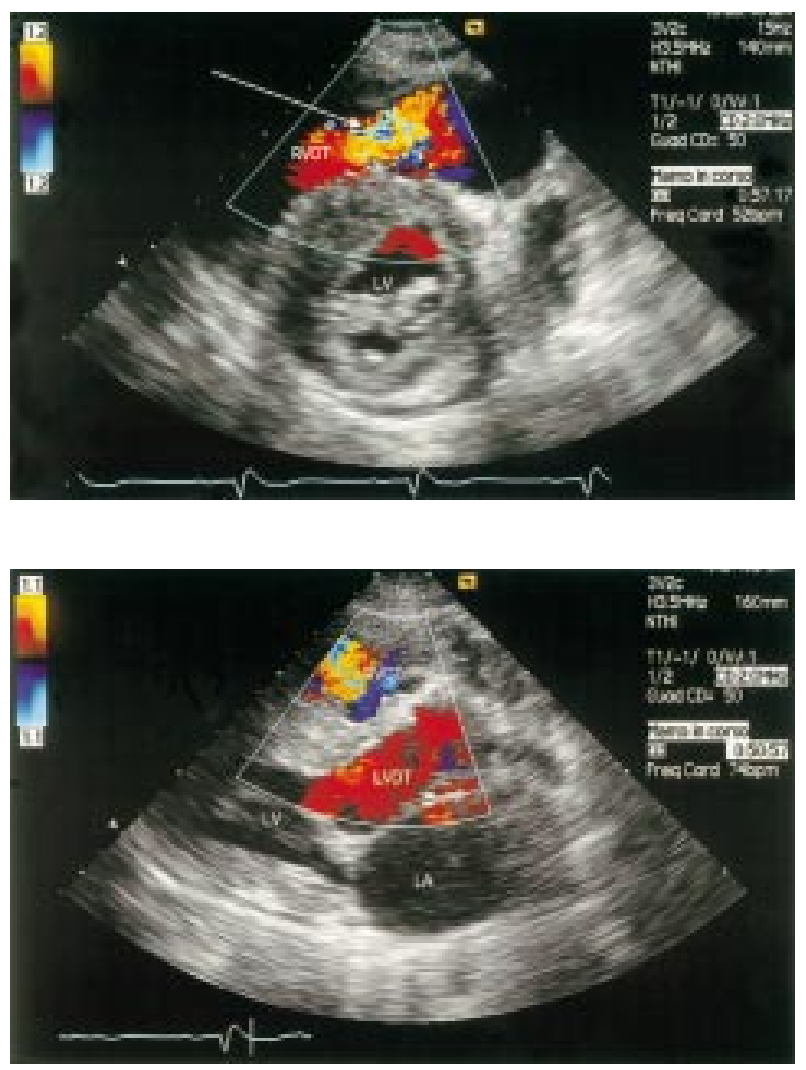

F Chirillo

R Zecchel

P Stritoni

fchirillo@ulss.tv.it 